\title{
Minimax: Portfolio Choice Based on Pessimistic Decision Making
}

\author{
Steffen Schaarschmidt ${ }^{1} \&$ Peter Schanbacher ${ }^{1}$ \\ ${ }^{1}$ Department of Economics, University of Konstanz, Germany \\ Correspondence: Steffen Schaarschmidt, Department of Economics, University of Konstanz, Universitätsstraße \\ 10, 78464 Konstanz, Germany. E-mail: steffen.schaarschmidt@uni-konstanz.de
}

Received: April 28, 2014

Accepted: May 26, 2014

Online Published: July 25, 2014

doi:10.5539/ijef.v6n8p23

URL: http://dx.doi.org/10.5539/ijef.v6n8p23

\begin{abstract}
We propose a fund allocation strategy for a highly risk-averse investor based on pessimistic decision making to construct portfolios of four major asset classes. Using US data (indexes of stocks, bonds, real estate, and commodities) from January 1990 to December 2010, we find that the proposed Minimax strategy performs well out-of-sample with respect to standard risk measures. Its performance is better than common alternative trading strategies such as fixed weights, minimum variance, or mean-variance methods. Portfolio weights are stable across time, resulting in lower turnover than any mean-variance related strategy. Finally, we find that optimal portfolios are widely diversified across all asset classes. This study suggests that the proposed Minimax strategy is implementable in portfolio management, and of special importance for investors with daily risk management.
\end{abstract}

Keywords: minimax, maximin, portfolio selection, portfolio management, portfolio choice

\section{Introduction}

Taking risk is usually accompanied by obtaining economic gains. In portfolio management, an investor typically trades risk against profits, according to his preference. However, in this trade-off it is fundamental to define "risk" appropriately. In the typical mean-variance model of Markowitz (1952), risk is defined as variation in returns. In this paper, we deviate from classical risk measures and we define risk in terms of a worst case scenario for two reasons. First, symmetric risk measures incorporate undesirable properties. Positive deviations of returns from their means should not be considered as risk, as volatility implies. Second, considering risk as measured by worst case scenarios is seasonable given recent periods of economic turmoil. During periods of moderate economic changes, risk measures such as volatility or Value-at-Risk may be quite effective. In extreme economic periods however, an investor may prefer to be much more conservative. For both reasons, we consider the highest realized loss to be the risk that matters most for an investor and consequently, we apply this risk measure for portfolio optimization.

Turning to first moments of return distributions, it has been shown in the past that estimating sample means suffers from serious problems. Slightly different mean estimates of asset returns can result in completely adverse portfolio positions. Literature proposes different ways to handle this estimation error. Minimax optimization not only implements a more appropriate risk measure for risk averse investors, but also avoids the major problem of estimating means.

A typical investor that may apply the Minimax trading strategy is a corporation, a pension fund, or a bank that invests in several asset classes and that is due to high regulatory requirements such as Basel II and Basel III. This regulation may require daily risk management, according to standard risk measures such as the Value-at-Risk. Corporations and funds that obey such regulation fear to exceed the risk limit of their portfolio on too many days per year, implying higher capital requirements. Consequently, the Minimax strategy meets the needs of institutional investors that aim at minimizing daily investment losses.

The second type of investor that needs to mimimize daily investment losses is an investor that is due to mark-to-market accounting, either for regulatory issues or, e.g., because of leveraged investment postitions that may trigger margin calls if the portfolio falls short of a certain level.

In order to find an "optimal portfolio" that satisfies any risk-averse investor, different portfolio selection rules have been proposed by a large body of finance research. To be mentioned first, the naive $1 / \mathrm{N}$ allocation rule goes back to the fourth century and survived until today. With this simple rule of thumb, one can obtain a well-diversified and risk reducing portfolio without having to estimate any model parameter. It is still a plausible 
and "hard to beat" benchmark for newly proposed portfolio selection methods. DeMiguel et al. (2009) state and show that there are still "miles to go" to beat the 1/N strategy, in particular for sample-based mean-variance strategies.

More and more of sophisticated asset allocation rules have been proposed over time. Markowitz (1952) derived the mean-variance theory, and still today, portfolio optimization based on the first two moments of the return is widely used. However, it suffers from serious caveats. First, it assumes either that the investor has an approximately quadratic utility function or that returns that are normally distributed and the investor's utility function is exponential. Second, estimating moments via their sample analogues leads to extreme and fluctuating weights, and as a result, portfolios perform poorly out-of-sample, in particular when trading costs are considered or when return distributions deviate from normality; see, for instance, Michaud (1998). A large literature examines extensions of the popular mean-variance model to reduce estimation error, for instance the Bayesian approach. In such a framework, Kan and Zhou (2007) combine mean-variance, minimum variance portfolios as well as the risk-free asset in order to decrease the influence of estimation error on investor's utility. Additionally, De Miguel et al. (2009a) show that imposing L2 norm constraints is equivalent to shrinking the covariance matrix. Lasso constraints are analyzed by Fan et al. (2012). For a summary of extensions to the mean-variance model, see DeMiguel et al. (2009b). We want to propose a trading strategy that fits any highly risk-averse investor, and to the best of our knowledge, we are the first to examine the performance of the Minimax strategy in a multi-asset-class portfolio choice context.

The Minimax strategy is a "pessimistic" trading strategy, because it chooses portfolio weights such that portfolio payoff is maximized in the worst case scenario. In particular, we use one year of daily returns, and we run a Monte Carlo simulation in order to model different states of nature for all possible portfolios. Using the worst case scenario, i.e. the state of nature with the lowest portfolio return, we choose portfolio weights such that portfolio payoff is maximal. Then we use the optimal weights for the next period, and so forth.

The first one that introduces the term "Minimax" in a portfolio context is Young (1998). He suggests linear programming for maximizing the minimum return of a portfolio based on historical returns, given a certain required minimum return. There have been earlier studies by, e.g., Sengupta (1982) and Lintner and Krasker (1982) on minimax optimization in portfolio choice. However, Young (1998) is the first to find that the Minimax model outperforms traditional mean-variance portfolios under a log-normal return distribution, and that it is compatible with expected utility maximization. There is a large body of research on linear programming models such as the Minimax model, and Mansini et al. (2003) provide a systemtatic overview as well as a discussion of their properties. Ding (2006) considers Minimax models without explicitly requiring a minimum return, as we will do in this paper.

This literature also connects to ambiguity aversion, another branch of Finance research. Gilboa and Schmeidler (1989) model situations where a decision maker has not enough information to assume one single prior distribution and determines investor preference as utility function over a set of multiple prior distributions. Chateauneuf et al. (2005) derive the theoretical framework for some important applications of multiple priors. In a recent paper, Garlappi et al. (2007) use confidence intervals for expected returns to model decision-making under multiple priors. They also show that if ambiguity aversion goes to infinity, the resulting portfolio is the global minimum variance portfolio. We will see later that the minimum variance portfolio is the hardest benchmark to beat in terms of portfolio risk and portfolio performance. The closest study to ours is Tütüncü and Koenig (2004). They use uncertainty sets for the moments of returns to obtain portfolios with best worst case behavior. Still, in their numerical exercise, they only examine equity and fixed income securities. We contribute to the literature by showing that Minimax portfolio choice strategies are valid and practically implementable for a representative multi asset investor, and we compare the performance of our Minimax trading strategy to common alternative benchmarks.

We use US stock, bond, real estate and commodity indexes to construct portfolios with yearly portfolio holding periods. Our representative investor is a multi asset investor that is due to stringent risk reporting-such as a large pension fund-and thus, cares about daily investment losses. The largest stake of US pension funds is invested in stocks and bonds. Yet, many funds diversify into real estate, commodities and other alternative investments as well. Looking at the Public Fund Survey (2009), we find that a representative US pension fund invested about $60 \%$ of its assets into stocks, about $20 \%$ into bonds, and the remaining part in other assets, such as real estate, private equity, hedge funds and commodities. Additionally, Belousova and Dorfleitner (2012) find that commodities add significant diversification potential to a portfolio of European stocks and bonds, and commodities can be particularly benefitial for risk-minimizing portfolio strategies. In order to compare our results to the US pension fund industry, we build a fixed weight benchmark that mimics a constant investment style of a representative US 
pension fund. Of course, we also test our results against the naive diversification strategy, which invests one fourth of funds into each asset class. In addition, we construct benchmarks that are based on common alternative portfolio selection concepts: Mean-variance and minimum variance optimization.

In our empirical analysis, we find outperformance of our Minimax-based portfolios against all other benchmarks considered. From a practical perspective, it is important to note that the weights of our Minimax trading strategy are stable over time and consequently, our Minimax strategy has a lower turnover than all mean-variance related strategies, even in the presence of short-selling constraints. This remark is noteworthy, because Minimax optimization leads to positive weights per definition, which is an appreciable feature from the viewpoint of a practitioner. Furthermore, the resulting Minimax portfolios are diversified across all considered asset classes with on average $11 \%$ invested in stocks, $54 \%$ invested in bonds, $15 \%$ invested in real estate and $15 \%$ in commodities, respectively.

The paper is organized as follows. Section 2 explains the Minimax decision and Section 3 introduces our database. Section 4 provides our empirical results and Section 5 provides robustness checks for the results. Finally, Section 6 concludes.

\section{Portfolio Selection Based on Minimax Decision Rule}

Minimax optimal portfolios provide the best worst-case behavior. For a certain year with $\mathrm{T}$ trading days, let there be $\mathrm{n}$ risky assets with random daily returns $R_{t}^{(i)}(i=1, \ldots, n$ and $t=1, \ldots, T)$. Further we assume that the investor holds a fraction $\mathrm{w}_{\mathrm{i}}$ invested in each asset. The vector of weights $W=\left(w_{1}, \ldots, w_{\mathrm{n}}\right)^{\prime}$ should satisfy $\mathrm{W}^{\prime} \mathrm{e}=1$ (with $\mathrm{e}=$ $(1,1, \ldots, 1)^{\prime}$ being a $(n \times 1)$ vectors of ones), i.e. all weights should add up to one. In the following, we also assume that short-selling is not allowed ( $w_{i} \geq 0$ for all $i$ ). This assumption is not problematic, since our representative investor is due to regulatory constraints, such that short-selling is not allowed for pension funds. Still, we relax this assumtion later on, and we examine the performance of all trading strategies in the presence of possible short-selling.

Let $W^{\prime} R_{t}=\sum_{i}=l^{n} w_{i} R_{t}^{(i)}$ be the portfolio payoff at day $\mathrm{t}$, given the asset return vector $R_{t}=\left(R_{t}^{(I)}, \ldots, R_{t}^{(n)}\right)^{\prime}$ and a certain asset allocation W. To determine suitable weights for time t, a risk averse investor may solve the following Minimax optimization problem:

(1) $\mathrm{W}^{*}=\operatorname{argmax} \mathrm{W} \min \tau \in\{\mathrm{t}-1, \ldots, \mathrm{t}-\mathrm{T}\} \mathrm{W}^{\prime} \mathrm{R}_{\tau}$ subject to $W^{\prime} e=1$ and $w_{i} \geq 0$. He considers the past $\mathrm{T}$ returns. In our application we set $\mathrm{T}=250$ to estimate the Minimax weights based on one year of daily returns. For any given asset allocation $\mathrm{W}$ there is a worst daily outcome of $\mathrm{W}^{\prime} \mathrm{R} \tau$ with $\tau \in\{\mathrm{t}-1, \ldots, \mathrm{t}-\mathrm{T}\}$. The Minimax portfolio is the asset allocation $\mathrm{W}^{*}$ which corresponds to the maximum return (or smallest loss) in the class of minimum outcomes.

\subsection{Intuition}

To make Minimax optimizations more intuitive, in the following we want to show how the Minimax procedure works with only two asset classes. If we look at the upper panel of Figure 1, we can see the possible portfolio return outcome at one specific day. All possible portfolio allocations are located on the straight line. Either we put all invested funds into stocks, or we invest all funds into bonds, or we spread the funds across both assets. At this particular day, stock performance was better than bond performance. Now we look at another possible historical trading day (middle panel of Figure 1), and we see that at this particular trading day, stock performance was worse than bond performance. Now consider first the two extreme positions, being completely invested in stocks and being completely invested in bonds. If one is completely invested in stocks, the worst outcome occurs in the second day and corresponds to a gross return of about 0.85 . One can improve this worst outcome by investing more into bonds. The worst outcome holding only bonds occurs in the first day with a gross return of 0.75. Again, one would be better off holding a more diversified portfolio. To sum up, in this two asset example Minimax optimization would take the asset allocation that corresponds to the intersection of the two lines.

In the last panel of Figure 1, we now see all possible portfolio allocations for the last 250 trading days. For any given asset allocation the worst outcome corresponds to the point with the lines closest to the point of origin. Going again from the extreme portfolios towards more diversified portfolios (from both sides), we end up in the scenario that yields the highest portfolio return, given the worst case scenario. In our case the Minimax portfolio is the asset allocation which corresponds to the returns at the kink, i.e. at about the coordinates $(0.65 / 0.25)$. We find that excluding one single day would not alter the optimal weights strongly. The Minimax leads to stable weights even though it depends on the single worst outcome. The optimization procedure is repeated each year to determine the weights for the following year. 

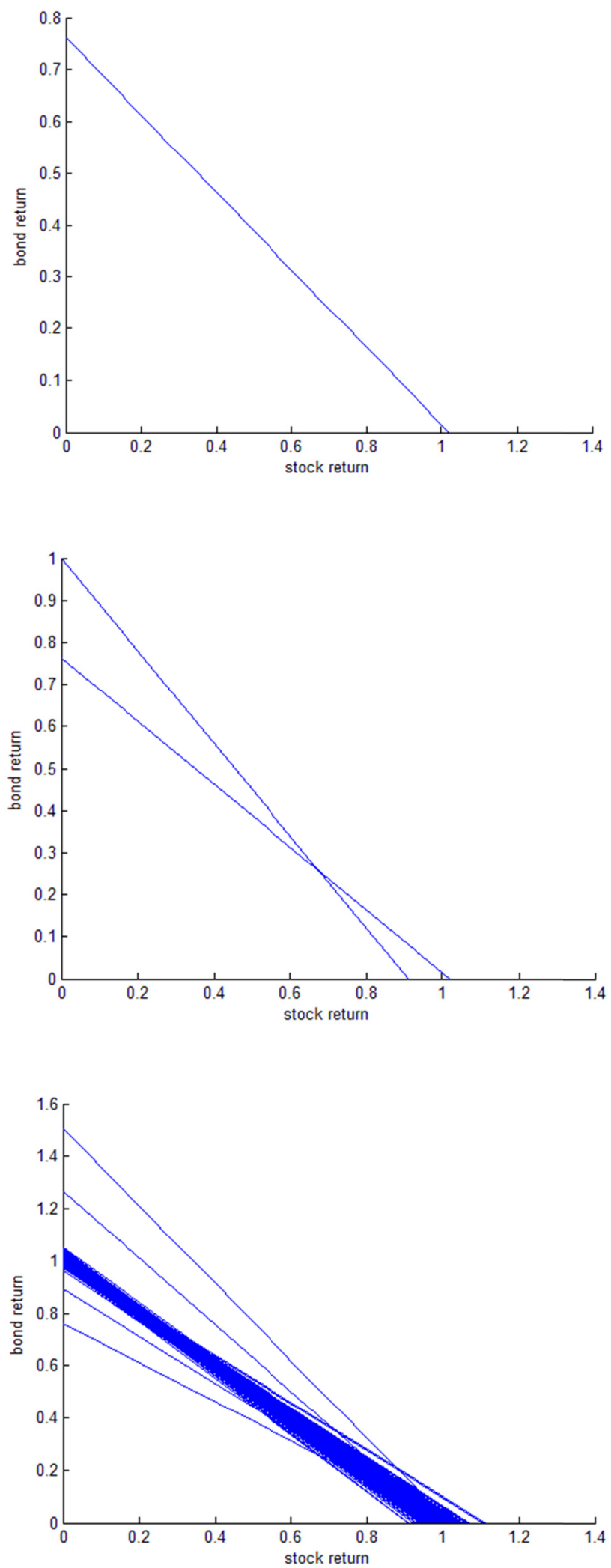

Figure 1. Illustration of minimax for two asset classes 


\subsection{Relationship to Minimum Variance Portfolio}

Consider a return time series of length $T,\left\{r_{t}\right\}_{t=1}{ }^{T}$ where all returns follow the same distribution. The expected minimum is given by the corresponding quantile of the distribution, i.e. $q_{\alpha}$ with $\alpha=1 / T$. Assume the returns follow a distribution such that the quantile can be expressed by $q_{\alpha}=\mu-z_{\alpha} \sigma_{\alpha}$. This holds amongst others for the $t$ - distribution. Now consider a combination of $n$ returns $W^{\prime} R_{t}$ with $R_{t}=\left(r_{t}^{(1)}, \ldots, r_{t}^{(n)}\right)$, mean $\mu=\left(\mu^{(1)}, \ldots, \mu^{(n)}\right)$ and covariance matrix $\Sigma$. The quantile of the combination is given by

(2) $q_{\alpha}(W)=W^{\prime} \mu-z_{\alpha} \sqrt{ } W^{\prime} \Sigma W$

If the investor applies the Minimum variance portfolio he supposes that the returns have a similar magnitude, i.e. $\mu^{(i)}=\mu^{(j)}$. Under this assumption we find that the Minimax optimization is equivalent to the minimum variance optimization, i.e.

(3) $W^{\star}=\operatorname{argmax}_{W, W^{\prime}=1} q_{\alpha}(W)$

(4) $=\operatorname{argmax}_{W, W_{e}^{\prime}=1} W^{\prime} \mu-z_{\alpha} \sqrt{W^{\prime} \Sigma} W$

(5) $=\operatorname{argmax}_{W, W_{e}^{\prime}=1} \mu-z_{\alpha} \sqrt{ } W^{\prime} \Sigma W$

(6) $=\operatorname{argmin}_{W, W^{\prime}=1} \sqrt{ } W^{\prime} \Sigma W$

We have seen that for similar means and a "nice" distribution of the returns the Minimax is similar to the minimum variance portfolio.

But why should an investor apply the Minimax instead of minimum variance portfolio? Mainly there are two advantages. Firstly, the Minimax asset allocation model adjusts quickly to structural shocks. Secondly, it implicitly considers the mean and not only the variance. We present the behavior by a structural shock to (i) the mean and to (ii) the variance. The reaction is shown in Figure 2. We consider two assets with daily returns $R_{t}^{(1)}$, $R_{t}^{(2)}$. In our simulation the uncorrelated normal returns have means $\mu^{(1)}=\mu^{(2)}=10^{-3}$ and variances $\sigma^{(1)}=\sigma^{(2)}=$ $10^{-5}$. The weights are estimated based on an estimation window of one year. As the returns are equal optimal weights are given by $W^{*}=(0.5,0.5)^{\prime}$. In the period from year 1 to year 2 the Minimax as well as the minimum variance portfolio are close to the optimal weights. The top plot of Figure 2 shows the reaction of a large shock to the mean. Other things equal, at year 2 the mean return of asset 1 drops to $\mu_{t}^{(1)}=-5 \cdot 10^{-3}, t \geq 2$. We observe that the Minimax weight in asset 1 is immediately decreasing. After less than 4 month (about year 2.3) the Minimax is only invested in asset 2 . The minimum variance portfolio decreases slowly its share in asset 1 . Note that the decrease does not stem from the fact that the variance has changed (it has not) but from the bias in the mean estimate. At period $t=2.5$ half of the returns of the estimation window have mean $\mu_{t}^{(1)}=10^{-3}(t \in(1.5,2))$ while the other half of the returns have mean $\mu_{t}^{(1)}=-5 \cdot 10^{-3}(t \in[2,2.5))$. The sample variance is high and the minimum variance portfolio invests less in asset 1 . As the estimation window is one year, at $t \geq 3$ there is no more bias and the minimum variance allocation is again the initial allocation: $W=(0.5,0.5)^{\prime}$. The Minimax optimization keeps excluding asset 1 . At $t \geq 2$ asset 1 is $N\left(-5 \cdot 10^{-3}, 10^{-5}\right)$ distributed and asset 2 is $N\left(10^{-3}, 10\right.$ ${ }^{-5}$ ) distributed. The weights of the Minimax are hence more reasonable.

The lower plot of figure 2 shows the behavior if a shock to the variance occurs. The returns are distributed as before, i.e. $R_{t}^{(i)} \sim N\left(10^{-3}, 10^{-5}\right)$ for $i \in\{1,2\}$. At year 2 , a shock to the variance of asset 2 occurs, i.e. $\sigma_{t}^{(2)}=$ $2 \cdot 10^{-5}$ for $t \geq 2$. Just before year 2 the Minimax portfolio readjusts from 0.55 to 0.475 . About one month after the shock Minimax optimization reacts to the shock and decreases the weight in asset 2, i.e. $W=(0.65,0.35)^{\prime}$ for about $t \geq 2$.2. Due to the large estimation window the minimum variance portfolio adjusts only slowly. Not until $t \geq 3$ the minimum variance portfolio reaches its optimal weight of $W=(2 / 3,1 / 3)$.

We find that the Minimax reacts quicker to structural breaks and avoids misspecifications as it also considers the mean. 

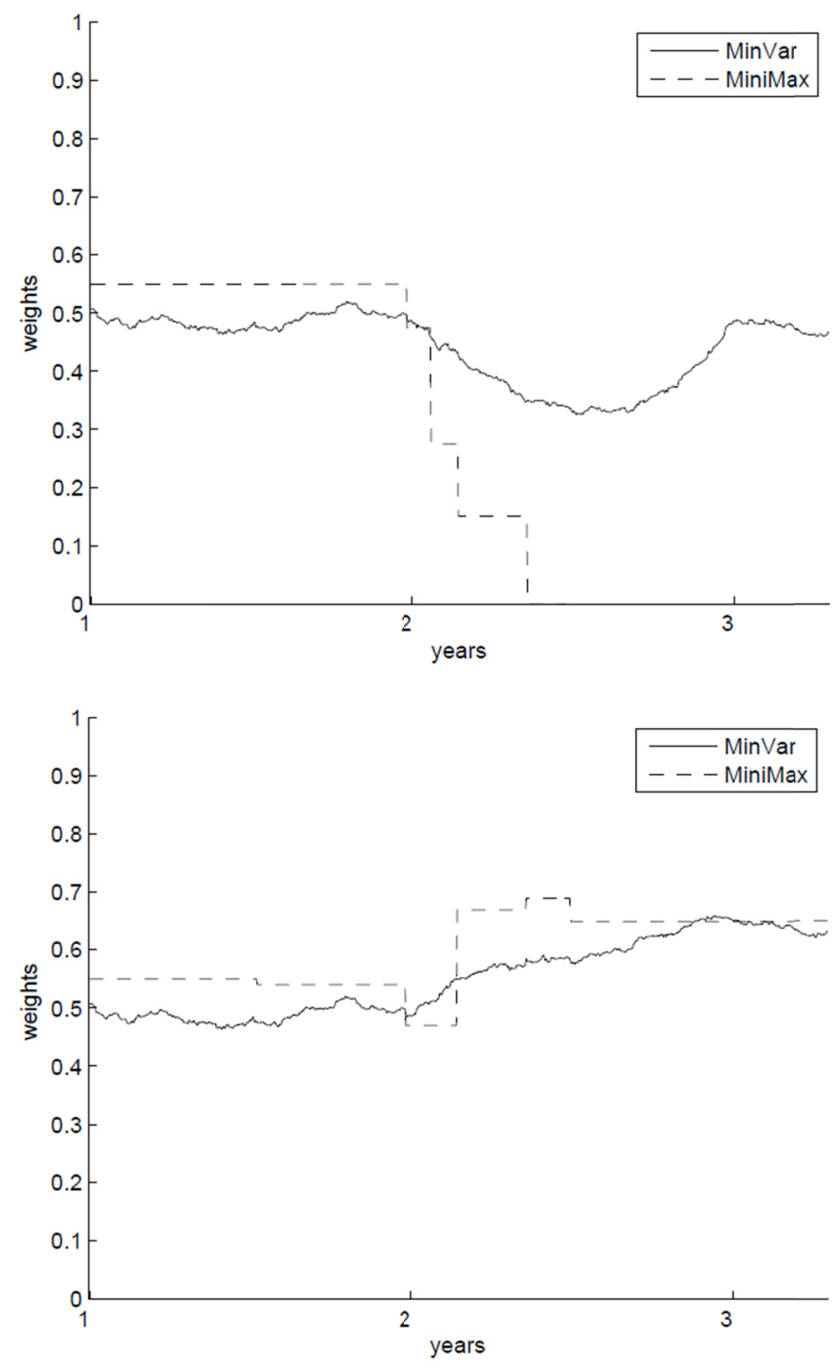

Figure 2. Structural shocks to mean and variance: minimax and minimum variance portfolios

The figure shows the weights of the Minimax and Minimum Variance (MinVar) portfolio under a structural shock to the mean (top plot) and the variance (lower plot) at $t=2$. The $y$-axis represents the weight for asset 1 (the weight for asset 2 is hence given by $1-y$ ). The $x$ - axis denotes the time (in years). The returns of both assets $i \in\{1,2\}$ are $N\left(\mu^{(i)}, \sigma^{(i)}\right)$ distributed with $\mu^{(i)}=10^{-3}$ and $\sigma^{(i)}=10^{-5}$. At year 2 there is a lasting shift of the mean $\mu^{(1)}=-5 \cdot 10^{-3}$ (top plot) and the variance $\sigma^{(2)}=$ $2 \cdot 10^{-5}$ (lower plot), respectively.

\subsection{Benchmark Models for Minimax Trading Strategy}

We test several benchmark models to compete against the proposed Minimax trading strategy. The benchmark models are chosen according to their relevance in the literature. We additionally show results for the "fixed weights" strategy, in order to mimick a representative investor's trading strategy. This fixed weights strategy is based on average holdings of US pension funds and invests $60 \%$ into stocks, $20 \%$ into bonds, $10 \%$ into real estate and $10 \%$ into commodities, respectively.

A strategy that is closely related to the fixed weights strategy is the equal weights strategy. Here we apply the naive -rule of thumb to allocate funds to different asset classes. Since $n=4$ in our case, we invest $25 \%$ of total funds into each asset class every year. One advantage of both strategies is obvious: Transaction costs are negligible. And as DeMiguel et al. (2009) state, the naive -rule is still hard to beat for any optimization, since estimation error does not exist.

Now we introduce the mean-variance related trading strategies that we are going to test our model against in the following section. First of all, we introduce the minimum variance portfolio as the competitor that is most likely to beat the Minimax trading strategy for the following reasons. A body of literature is written on problems in 
mean-variance optimization due to estimation of expected returns. Small deviations of expected returns from realized returns result in high fluctuating weights. Jagannathan and Ma (2003) therefore propose to just ignore expected returns and use the minimum variance portfolio instead. It mimimizes the risk of a portfolio and therefore is a suitable strategy for any highly risk-averse investor. The main difference to Minimax is the risk measure, which is symmetric (volatility). In particular, the minimum variance optimization is given as

(7) $\min _{W} W^{\prime} \Sigma W$;

subject to $W^{\prime} e=1$ and $w_{i} \geq 0$, with $\Sigma$ beeing the $4 \times 4$ variance-covariance matrix of our four asset classes.

The last strategy that we want to test our Minimax strategy against is the mean-variance strategy. It is based on Markowitz (1952) and relates risk and return as given in the following optimization:

(8) $\max _{W} W^{\prime} \mu-\lambda W^{\prime} \Sigma W$;

subject to $W^{\prime} e=1$ and $w_{i} \geq 0$. We calculate mean-variance optimal portfolios based on a risk-aversion parameter of three, i.e. $\lambda=3$ (as commonly chosen, see e.g., Kan \& Zhou, 2007).

\section{Data}

To form portfolios, we use indexes on four major asset classes that big US pension funds commonly and mainly invest in: stocks, bonds, real estate and commodities. We notice that a major share of pension funds actually invests in more asset classes (e.g., in hedge funds). We do only consider stocks, bonds, real estate and commodities for two reasons: First, the share invested in alternative asset classes is relatively small with about $5 \%$. Second, in our Minimax optimization, we are limited to a certain number of asset classes due to computational issues, and four asset classes turn out to be still feasible.

Our database is Thomson Datastream, and we download daily prices of performance indexes on the four asset classes. For stocks, we consider the total return (dividends included) on the S\&P 500 index. Bond performance is measured by the Barclays Aggregate Bond index, also in terms of a total return index. For real estate, we use the Datastream US real estate index, which is an appropriate proxy for the US real estate market. We note that pension funds also may directly own residential and commercial real estate. However, we lack daily prices and therefore rather consider the performance of a real estate investment trust, where daily valuation is available. Commodity market performance is proxied by the common used S\&P GSCI index, which is widely diversified across commodities. Finally, we take the yields on a 90-day Treasury Bill as a proxy for the risk-free rate.

Our indexes cover 21 years from January 1990 to December 2010, resulting in 5480 daily observations. We provide the performance of all asset classes in Figure 3 and descriptive statistics for all asset classes in Table 3.

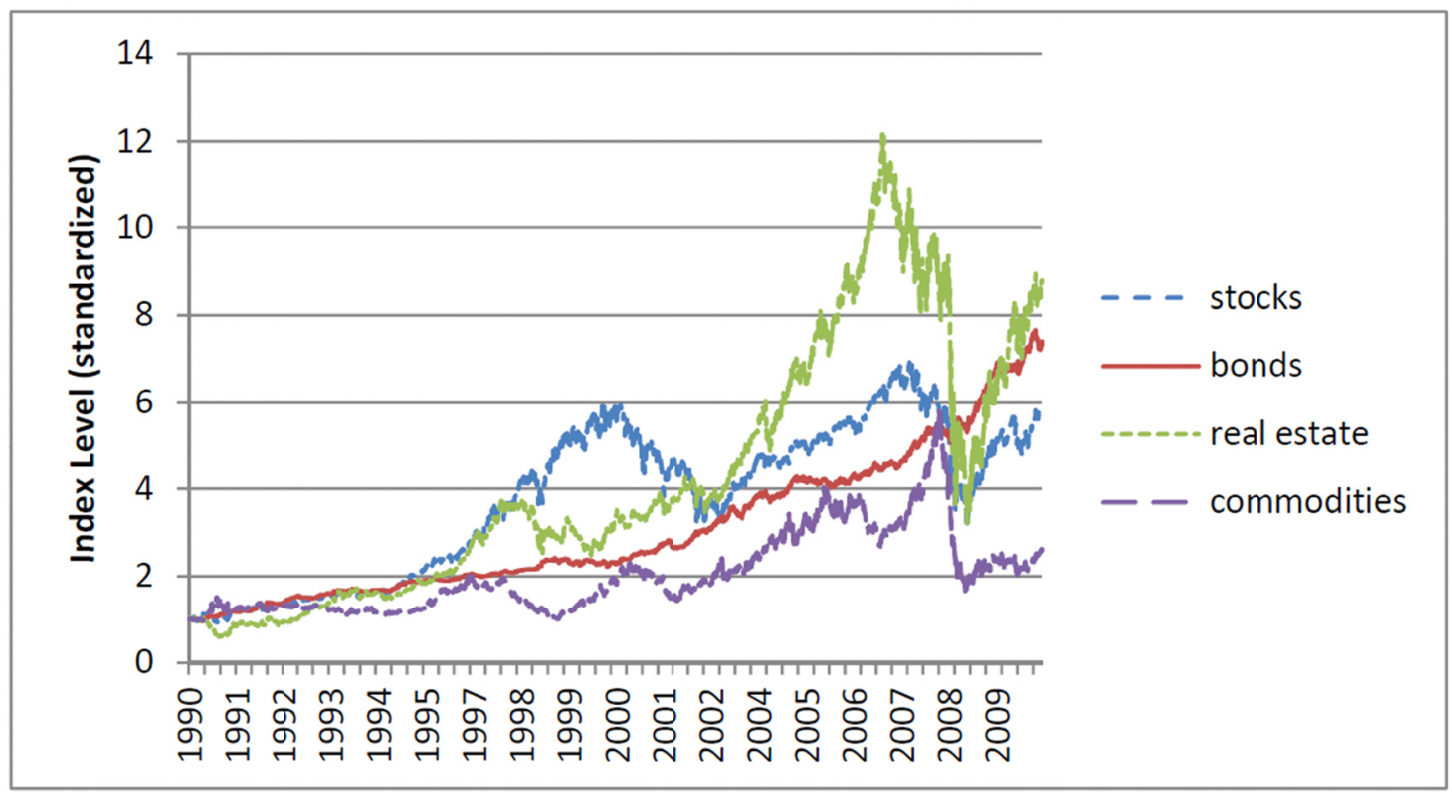

Figure 3. Performance of asset classes

Note. The figure shows the time series of one dollar invested into each asset class in January 1990. Sample period is January 1990 to December 2010. 
Table 3. Descriptive statistics

\begin{tabular}{llll}
\hline PERFORMANCE & Geometric Return (\%) & Volatility (\%) & Sharpe Ratio \\
\hline Stocks & 0.033 & 1.15 & 0.022 \\
Bonds & 0.037 & 0.43 & 0.055 \\
Real Estate & 0.040 & 1.66 & 0.024 \\
Commodities & 0.018 & 1.37 & 0.009 \\
\hline
\end{tabular}

\begin{tabular}{llllll}
\hline DISTRIBUTION & Geom. Return (\%) & Min (\%) & Max (\%) & Skewness & Kurtosis \\
\hline Stocks & 0.033 & -9.0 & 11.6 & -0.01 & 9.46 \\
Bonds & 0.037 & -4.0 & 5.1 & -0.66 & 8.29 \\
Real Estate & 0.040 & -18.3 & 18.6 & 0.48 & 23.53 \\
Commodities & 0.018 & -16.8 & 7.9 & -0.41 & 7.48 \\
\hline
\end{tabular}

\begin{tabular}{llll}
\hline CORRELATION & Stocks & Bonds & Real Estate \\
\hline Bonds & -0.07 & & \\
Real Estate & 0.65 & -0.01 & \\
Commodities & 0.11 & 0.03 & 0.09 \\
\hline
\end{tabular}

All numbers are based on daily returns. Returns (\%) are calculated geometrically. The Sharpe Ratio is calculated by substracting the daily risk-free rate from daily returns, and then dividing by their daily standard deviation. Min (\%)/Max (\%) is the minimum return/maximum return of all realizations in the sample period, denoted in percentage. Correlations are caculated unconditionally on the returns. All measures are calculated over the whole period of 1990 to 2010.

We note that in the considered time period, real estate has been the asset class with the highest return, but also with the highest volatility. Especially during the financial crisis in 2008 we notice a heavy decline of US house prices. Also stock and commodity prices decreased during that time, in contrast to US bonds, which incorporate the lowest volatility of all four asset classes. Their volatility is only as high as $0.43 \%$ for daily prices, which is by far less than half of the volatility of stocks, real estate and commodities. Another risk measure, minimum return, indicates that bonds are less risky than stocks, real estate and commodities, losing only $4.0 \%$ in the worst trading day over the whole period. Bonds are also attractive, when we relate risk and return, looking at the Sharpe Ratio. Again, bonds outperform other asset classes. We can thus expect that bonds will play a major role in all risk-reducing optimizations.

When we look at correlations between asset classes, we find that almost all asset classes exhibit low correlations. The only exception is a relatively high correlation between stocks and real estate. We conclude that this is because our real estate investment trust (REIT) is exchange-traded. We can thus further expect that a risk-reducing optimization will diversify funds across asset classes, since diversification potential is high due to low asset correlations.

\section{Empirical Results}

We now show results for our empirical analysis. Each year in January, we run the Minimax and mean-variance optimizations based on the last 250 trading days. We obtain optimal weights for each optimization strategy, and we hold a portfolio based on these optimal weights for one subsequent year. After this year, we evaluate the (out-of-sample) performance and, we rerun the optimization based on return realizations based on that particular year, and so on. Furthermore, we show results for the equal weights and fixed weights strategies, which merely reallocate funds to their predetermined shares. Table 4 presents results of performance and risk for all trading strategies.

Looking at the out-of-sample performance of our portfolios, we can see that the mean-variance portfolio (with $\lambda$ $=3$ ) yields the highest return, but also the highest volatility. Neglecting the mean-variance portfolio, with an average daily return of $0.05 \%$ and a volatility of $0.54 \%$, the Minimax strategy has the highest Sharpe Ratio. We can also see that the minimum variance portfolio has the lowest (out-of-sample) volatility, which is in line with the objective of minimum variance optimization, i.e., to minimize (in-sample) volatility. Interestingly, by diversifying funds widely across all asset classes, the naive strategies beat the mean-variance portfolio in terms of volatility, still the Minimax and the Minimum Variance strategies outperform naive diversification strategies not only in terms of risk, but even yield a higher return. All portfolios have lower Sharpe Ratios with respect to 
Minimax (but mean-variance), and the difference of Sharpe Ratios with respect to Minimax is significant for all portfolios, except for minimum variance. All results are obtained by imposing short-selling constraints, which implicitely enhance the performance of mean-variance related competitors; see, for example, Jagannathan and Ma (2003).

Table 4. Performance and risk results

\begin{tabular}{lllllll}
\hline PERFORMANCE & Return (\%) & Vola (\%) & Sharpe Ratio & Excess Return & Turnover & CEV3 \\
\hline Minimax & 0.052 & 0.54 & 0.071 & over Minimax (\%) & 9.2 & 4.8 \\
Minimum Variance & 0.039 & 0.40 & 0.063 & -0.013 & 9.9 & $3.7^{* * *}$ \\
Equal Weights & 0.040 & 0.78 & $0.032^{* * *}$ & -0.012 & 0 & $3.0^{* *}$ \\
Fixed Weights & 0.040 & 0.77 & $0.034^{* * *}$ & -0.012 & 0 & $3.1^{* *}$ \\
Mean-Variance & 0.105 & 0.97 & $0.094^{* * *}$ & 0.053 & 26.4 & $9.1^{* * *}$ \\
\hline
\end{tabular}

\begin{tabular}{lllll}
\hline RISK & Volatility (\%) & $\operatorname{VaR}(0.95)(\%)$ & $\operatorname{VaR}(0.99)(\%)$ & Max. Loss $(\%)$ \\
\hline Minimax & 0.54 & -0.57 & -0.99 & -2.06 \\
Minimum Variance & 0.40 & -0.54 & -1.08 & -2.63 \\
Equal Weights & 0.78 & -0.95 & -2.29 & -8.71 \\
Fixed Weights & 0.77 & -0.99 & -2.07 & -7.31 \\
Mean-Variance & 0.97 & -1.71 & -3.59 & -9.25 \\
\hline
\end{tabular}

All numbers are based on daily returns. Return (\%) is calculated geometrically. The Sharpe Ratio is calculated by substracting the daily risk-free rate from daily returns, and then dividing by their daily standard deviation. Turnover is proxied by the sum of absolute deviations in weights before and after rebalancing. We rebalance portfolios every year, using past 250 trading days for our optimizations. CEV3 is the Certainty Equivalent for a mean-variance investor with risk aversion parameter $\lambda=3$. VaR stands for Value-at-Risk, which indicates the minimum return (or equivalently, the maximum loss) for a certain significance level ( $95 \%$ or $99 \%$ ). Based on the test of Ledoit and Wolf (2008) significant Sharpe Ratio and CEV3 differences of the alternative portfolio model and the Minimax at 1\%, 5\%, 10\% level are denoted by ***,** and *, respectively. Short-selling is not allowed for all optimizations. All measures are calculated over the whole period of 1990 to 2010 .

However, we have to bear in mind that this gain comes at the cost of rebalancing the portfolio from year to year. Active rebalancing leads to higher transaction costs, which may compensate the benefits of optimization and which works in favor of easy rule-of-thumb strategies with low portfolio turnover. The strategies with less turnover are by construction the equal weights and fixed weights strategies, which cause almost no transaction costs. We proxy turnover by the sum of total deviation in weights from one period to another:

(9) $T O_{t}=\sum_{i=1}{ }^{4}\left|w_{i, t}-w_{i, t-1}\right|$;

Yet, we neglect here that asset prices change over time, so even naive strategies have slightly positive transaction costs, since portfolios have to be reset to their predetermined fixed allocation from year to year. Mean-variance portfolios have the highest turnover, which is more than 20 times as high as the turnover of minimum variance and Minimax strategies. This is not surprising, though. Literature shows that pure mean-variance related portfolio optimizations exhibit strongly fluctuating weights, which result in high transaction costs. Therefore, several approaches have been proposed to handle the problem of estimation error, which is mainly responsible for highly fluctuating weights. For instance, Ledoit and Wolf $(2003,2004)$ suggest to use a weighted average of the sample covariance and another estimator. By doing so, extreme weights can be prevented and thus, transaction costs can be substantially lowered. For practical implementation, transaction costs play a major role. Remember that our representative agent is a big US pension fund investing billions of dollars for its clients. A small reduction in costs is equivalent to saving a huge amount of money.

Now it is interesting to compare turnover of our Minimax approach to minimum variance optimization. We can see that turnover is comparable, but still, turnover is slightly less for the proposed Minimax strategy.

Last but not least, comparing the Certainty Equivalent of all strategies, we find that the Mean-Variance approach yields the highest CEV3 value out-of-sample, which is not surprising, too, since the Certainty Equivalent is a concept originating in the mean-variance theory of Markowitz (1952). Again, CEV3 differences of all portfolios with respect to Minimax are significant. To sum up the performance of our four portfolios, we find that Minimax is always best or second best, and thus, Minimax is very consistent across all performance measures used. Now 
we want to stress one component of performance, which is highly relevant for a big US pension fund portfolio, namely its risk. Risk can be measured differently, and here we use a selection of risk measures that is often used in scientific work as well as in practical work. We use volatility as a common symmetric risk measure in finance, we use the Value-at-Risk (VaR) measure as the most frequently used assymetric risk measure. Finally, we use the maximum loss as an asymmetric risk measure that fits best our risk aversion assumption, namely that our representative investor is highly risk-averse, and as a result, he fears high losses.

Having documented that minimum variance beats all competitors in terms of volatility, again, it is noteworthy that the Minimax approach yields not only a higher return than both naive asset allocation strategies, but additionally Minimax optimal portfolio returns exhibit much less volatility than returns of portfolios that could represent a typical US pension fund portfolio. We thus document an improvement both in return and in risk.

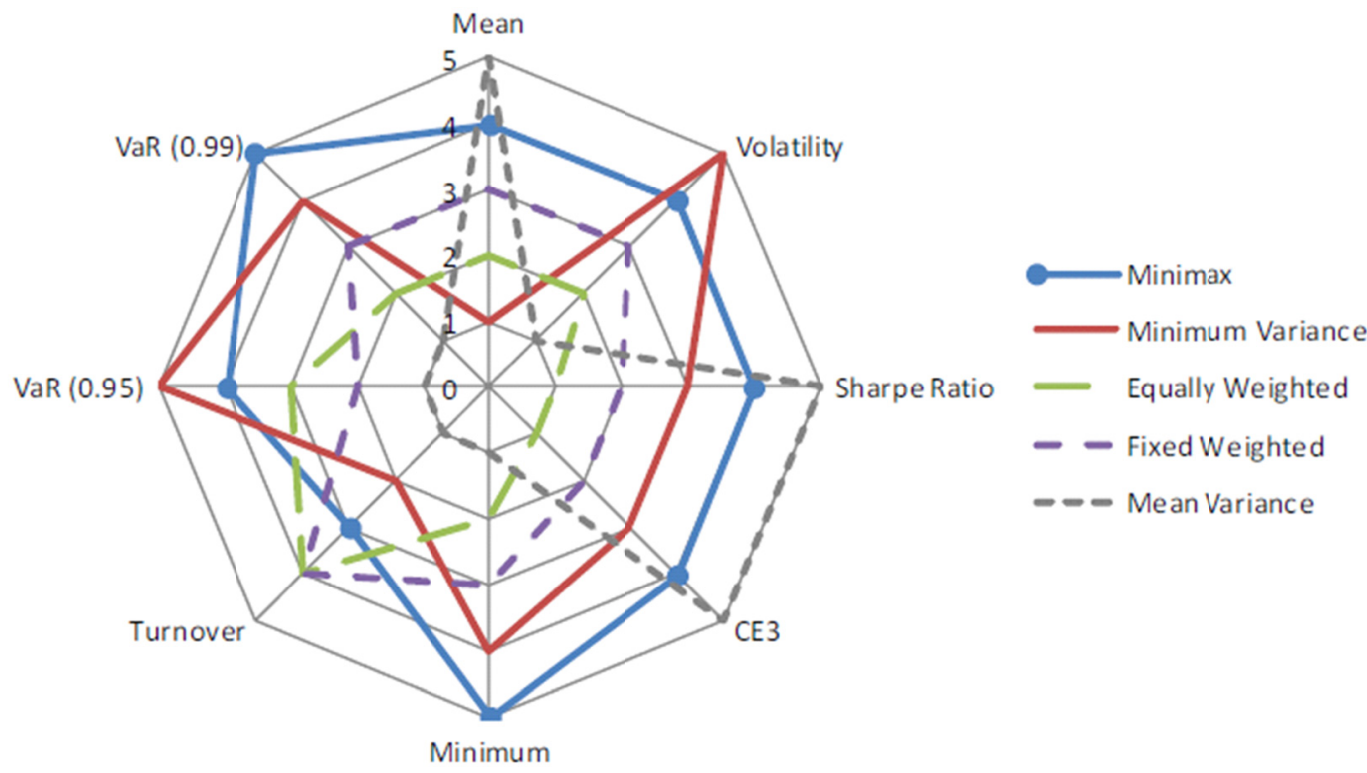

Figure 4. Graphical illustation of performance and risk results

All performance and risk results are based on the numbers and variables in Table 4. We rank all strategies, while 1 is given to the strategy that perfoms the poorest for a particular risk measure, and 5 is given to the best strategy, respectively. All measures are calculated over the whole period of 1990 to 2010.

The next risk analysis is based on the Value-at-Risk measure. It indicates the maximum loss that is possible for a given significance level, here we choose the level according to $95 \%$ and $99 \%$. We find that on the $95 \%$ significance level, the minimum variance portfolio is the one with the lowest maximum loss. Strategies of representative pension funds are exposed to risk, which is almost twice as high as minimum variance and Minimax optimizations, based on the $\operatorname{VaR}(0.95)$ risk measure. The results are basically the same when we look at the Value-at-Risk at the $99 \%$ significance level. Again, naive diversification strategies load twice as much risk than risk-minimizing strategies. The mean-variance portfolio is not competitive based on any risk measure. Interestingly, the minimum variance portfolios outperform Minimax portfolios at the $95 \%$ VaR significance level, but it appears to be the other way round at the $99 \%$ significance level. The same result is obtained for maximum loss, where the Minimax portfolio clearly dominates all other strategies. We can see from the results that for a highly risk-averse investor, minimum variance is the hardest competitor for our Minimax model. 


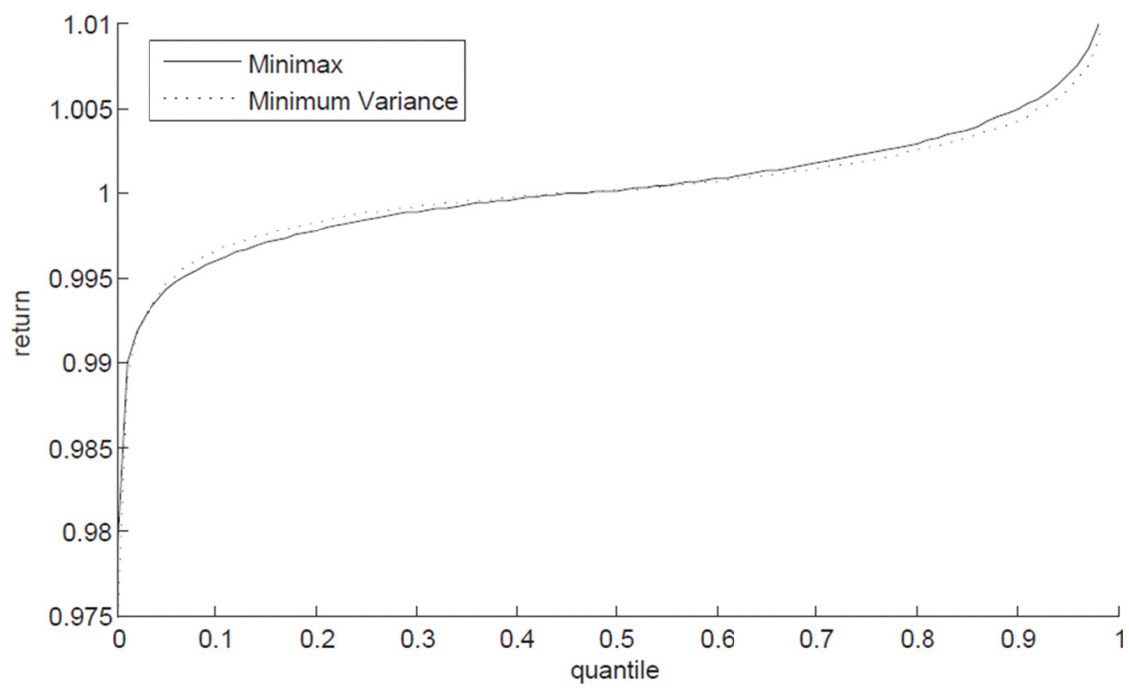

Figure 5. Empirical quantiles of minimax and minimum variance

The figure shows the portfolio return distributions of Minimax and minimum variance strategies. The abscissa denotes quantiles and the ordinate axis shows gross returns, where 1.00 stands for zero portfolio return. The portfolio returns are calculated over the whole period of 1990 to 2010.

The last result indicates that Minimax portfolios outperform its closest competitor in the very left tail of return distributions, i.e. for a highly risk-averse agent like ours. We want to examine this issue by looking at the return distributions of Minimax and minimum variance portfolios. Figure 5 shows both distributions. Looking at very low portfolio returns (i.e., at the very left hand of Figure 5), we notice that Minimax dominates minimum variance. In other words, for the portfolio risk that matters most for a highly risk-averse agent, Minimax outperforms the minimum variance strategy. For low, but not extreme returns we can see that it is the other way round, while turning to positive returns, again Minimax dominates minimum variance. This is because minimum variance minimizes a symmetric risk measure, volatility, and therefore chooses a portfolio with returns that do not deviate too far from their mean. This, however, is not a nice property. Imagine a portfolio A that generates highly volatile, but high returns and a portfolio B that generates lower and less volatile returns than portfolio A. The minimum variance strategy would always prefer portfolio A to B, although logically, a risk-averse investor with any reasonable utility function should prefer portfolio B to A (and this is what Minimax would do).

The outperformance of Minimax over minimum variance in terms of the common used Sharpe Ratio can thus be summarized as a dominance of Minimax in the right-hand part (positive returns) of return distributions as well as in the very left part (extreme losses).

Table 5. Portfolio characteristics

\begin{tabular}{lllllll}
\hline WEIGHTS & Minimum & Maximum & Average (Vola) & Average (Vola) & Average (Vola) & Average (Vola) \\
\hline All & All & Stocks & Bonds & Real Estate & Commodities & \\
Minimax & 0 & 0.90 & $0.11(0.11)$ & $0.54(0.24)$ & $0.15(0.18)$ & $0.15(0.11)$ \\
Minimum Variance & 0 & 0.92 & $0.15(0.10)$ & $0.61(0.26)$ & $0.09(0.14)$ & $0.10(0.10)$ \\
Equal Weights & 0.25 & 0.25 & $0.25(0)$ & $0.25(0)$ & $0.25(0)$ & $0.25(0)$ \\
Fixed Weights & 0.1 & 0.60 & $0.6(0)$ & $0.2(0)$ & $0.1(0)$ & $0.1(0)$ \\
Mean-Variance & 0 & 1.00 & $0.10(0.30)$ & $0.10(0.30)$ & $0.43(0.51)$ & $0.33(0.48)$ \\
\hline
\end{tabular}

\begin{tabular}{lllll}
\hline CORRELATION & Minimax & Minimum Variance & Equal Weights & Fixed Weights \\
\hline Minimum Variance & 0.73 & & & \\
Equal Weights & 0.54 & 0.52 & & \\
Fixed Weights & 0.50 & 0.52 & 0.52 & 0.24 \\
Mean-Variance & 0.25 & 0.24 & 0.78 & \\
\hline
\end{tabular}


All numbers in the upper table are based on asset weights resulting from portfolio otimizations. "Minimum All" and "Maximum All" denote minimum and maximum weights for any asset class, whereas "Average" denotes the average weight in one specific asset class. Correlation measures in the table below are again based on daily portfolio returns. All measures are calculated over the whole period of 1990 to 2010 .

\subsection{Analysis of Portfolio Weights}

Besides attractive performance characteristics, one should also look at other portfolio characteristics. A big pension fund aims at maintaining rather constant portfolio weights than at rebalancing frequently due to higher transaction costs. We already considered turnover as a proxy for transaction costs, and we found that the naive strategies cause the least transaction costs, Mean-Variance optimization leads to the highest transaction costs, and turnover of minimum variance and Minimax strategies is quite comparable.
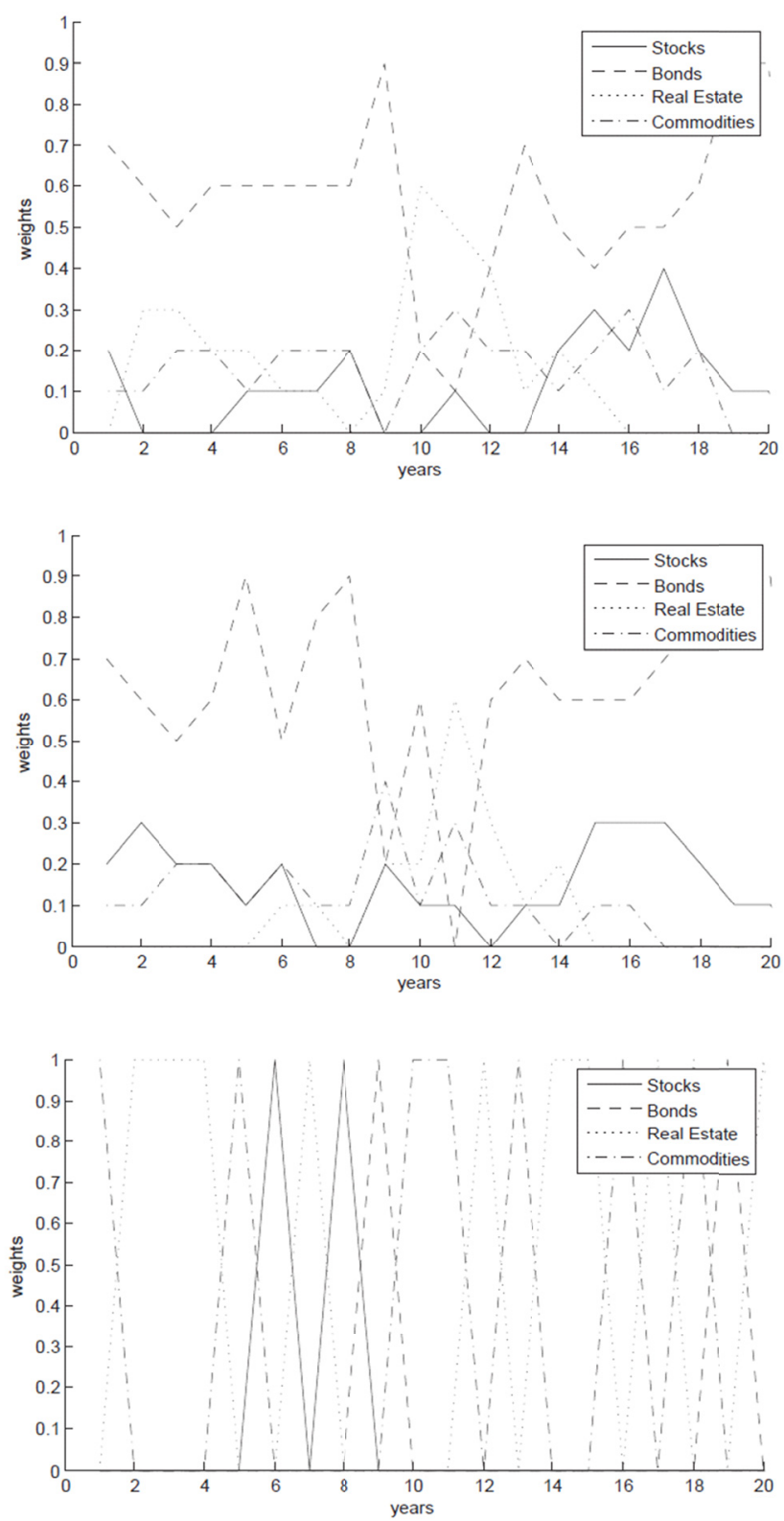

Figure 6. Time series of portfolio weights

This fact leads to the question, to what extent both best performing strategies are similar. When we consider correlations of portfolio returns, we can see that Minimax and minimum variance portfolio returns are highly 
positively correlated with a correlation coefficient of 0.73 . Only one other pair of portfolio strategies is as highly correlated. The high correlation indicates that the risk minimizing mechanisms must be similar, and it has also been shown theoretically that both strategies lead to almost the same optimal portfolio, if returns are normally distributed (see e.g. Young (1998), p. 675). Both Minimax and minimum variance hold the largest fraction of funds in bonds. We saw before that bonds have quite nice performance characteristics, they have the highest Sharpe Ratio and the lowest volatility. Thus, it is not surprising that, on average, a 54\% share of Minimax portfolios and a $61 \%$ share of minimum variance portfolios is invested into this asset class. In contrast, Minimax invests a little more into real estate $(15 \%)$ and commodities $(15 \%)$ than the minimum variance strategy $(9 \%$ and $10 \%$, respectively). The mean-variance strategy is on average heavily invested into real estate and commodities, while their share in stocks and bonds is only $20 \%$. This, however, is a result of fluctuating weights in the mean-variance optimization, as we can see in Figure 6. Mean-variance portfolios always consist of only one asset class, which is held for one period. In contrast, Minimax and minimum variance strategies seem to be quite stable over time. Figure 6 plots the time series of portfolio weights.

This figure shows portfolio weights for each asset class, resulting from Minimax, minimum variance and mean-variance optimizations. Rebalancing takes place every year. The number 1 on the abscissa corresponds to year 1991, since we need one year of historical returns (January 1990 to December 1990) for the optimizations.

\section{Robustness Checks}

In this section, we test our results for robustness. To do so, we slightly change Minimax as well as other optimization procedures, and we see that results basically stay the same.

First, we rerun optimizations and fixed weight strategies for yearly returns, in contrast to daily returns. We provide results for the main performance measures in Appendix A Table 6 shows that when we look at yearly returns, the minimum variance portfolio dominates Minimax both in terms of risk and return. This is due to the return distribution. On the one hand, yearly returns are closer to the normality assumptions than daily returns, and thus, Minimax optimization loses its advantage in the very left part of the return distribution, because high portfolio losses are rare. On the other hand, the closeness to normality of portfolio return works in favor of optimizations using volatility as a symmetric risk measure. However, we see that Minimax is able to beat naive asset allocation stategies and therefore, is preferable from the point of view of big US pension funds. Again, we want to highlight the advantage of Minimax for daily periods of interest, for instance for institutional investors that are due to daily risk reporting.

As a second robustness check, we want to consider a different rebalancing period. Up to now, we rebalance portfolios once a year, how it is commonly done in the literature, and our estimation window consists of 250 past observations. Now we want to consider a rolling windows approach: We rebalance every day, and again, the estimation window consists of 250 past observations. Results are provided in Appendix B shows that Minimax has the highest Sharpe Ratio. Again, and based on the Sharpe Ratio, we find outperformance of the Minimax portfolio selection over all considered benchmarks. For Minimax portfolio returns, volatility is slightly higher than for minimum variance portfolios, still returns are again higher. All other results stay qualitatively the same as in the base scenario with yearly rebalancing.

In a third robustness check, we allow for short-selling, i.e. we do not require the asset weights to be positive. This obviously works against mean-variance related optimizations that suffer from estimation error. We can see results for optimizations in Appendix $\mathrm{C}$ shows performance and risk characteristics for our standard case, daily returns and yearly rebalancing. For minimum variance portfolios, allowance for short-selling does not change. For mean-variance portfolios, however, we can see that the portfolio risk increases substantially, as well as turnover, which is a result of large long and short positions in the portfolio. Such a portfolio is not preferable for our typical investor, since it holds substantial risk, and turnover is around 25 times higher than for minimum variance or Minimax portfolios. However, the mean-variance portfolio without short-selling restriction yields a Sharpe Ratio which is higher than that of its competitors. Again, the Sharpe Ratio for our Minimax portfolio is higher than that of minimum variance. Table 8 provides robustness checks for daily returns and daily rebalancing without short-selling restrictions. As expected, turnover increases in addition for all portfolios and yields 3818.9 for the mean-variance portfolio, as opposed to around 40.4 and 46.5 for Minmax and minimum variance portfolios, respectively. Again, the Sharpe Ratio of Minimax exceeds the Sharpe Ratio of the mean-variance related strategies.

The last robustness check is with respect to crises periods. In Appendix D, we analyze the performance of the Minimax portfolio and its competitors during crises periods. We consider the following crises: (1) the Internet bubble during 2000/2001, (2) the Terrorist attack in 9/11/2001, (3) the Banking crises during 2007/2008 and 
subsequent (4) Economic recession during 2008-2010. The Minimax portfolio suffers of the smallest maximum loss in all crises but one. In all considered crises it has the largest or second largest CE3 performance. In all cases it achieves Sharpe ratio which is above average-sometimes best. We conclude that in particular for crises periods, the Minimax portfolio performs well.

\section{Conclusion}

In this paper, we propose a trading strategy called Minimax, which is based on pessimistic decision making and which suits a highly risk-averse investor. In particular, Minimax is an appropriate asset allocation optimization for big pension funds or other institutional investors that are due to daily risk reporting, either because of regulatory requirements or because of mark-to-market accounting. Maximizing the worst case payoff of a portfolio, Minimax strategies are practically easy to implement and constitute a proper alternative to common risk-minimizing optimizations such as minimum variance.

We use US data on indexes of stocks, bonds, real estate and commodities from January 1990 to December 2010 in order to calculate daily portfolio returns. We compare the proposed allocation strategy with alternative asset allocation strategies. Therefore, we calculate a minimum variance portfolio, which minimizes volatility within one year of historical daily returns, a mean-variance portfolio, an equal weights strategy, and a typical US pension fund portfolio, which imitates an asset allocation that a representative investor could possibly run.

Our main result is that the proposed Minimax strategy outperforms all competitors, in terms of different risk and performance measures. We find the minimum variance portfolio to be the hardest competitor for Minimax, assuming a highly risk-averse agent, and portfolio characteristics of both strategies are comparable. We show that the particular advantage of the Minimax strategy is the avoidance of very large losses. Optimizations based on volatility as a symmetric risk measure such as minimum variance strategies fail to provide optimal portfolios with attractive performance characteristics, because they minimize not only negative, but also positive returns. Minimax, however, only cares about huge portfolio losses, and provides preferable performance characteristics by allowing positive portfolio returns. Naive portfolio allocation rules are not competitive to Minimax in terms of performance and risk. Still, this does not mean that naive portfolio allocation strategies are in general not appropriate for any investor. Studies show that many optimization strategies fail to beat simple rules of thumb, and by diversifying funds across different asset classes, one can reduce risk efficiently without imposing strong ex ante restrictions. Another advantage of fixed weight strategies, besides their simplicity and applicability, is low turnover. This feature makes them particularly interesting for long-term investors that face high transaction costs. Mean-variance optimization is not competitive to all above mentioned strategies, due to high estimation error.

Considering portfolio characteristics, we find admirable features for portfolio weights that result from all strategies but from mean-variance optimization. Portfolio weights are relatively stable over time for Minimax and minimum variance, resulting in comparable turnover and transaction costs. Transaction costs are particularly high for the mean-variance portfolio, implied by high estimation error and extreme portfolio weights. Minimax and minimum variance portfolios both invest on average about $50-60 \%$ of their funds into bonds, while the rest of funds is spead across the remaining asset classes. By doing so, the resulting portfolios are satisfyingly diversified.

In a last analysis, we check our results for robustness. For yearly returns (instead of daily returns), we lose dominance over the minimum variance strategy. This is particularly due to the fact that yearly returns are "closer to normality" than daily returns. In a scenario with normally distributed returns, portfolios based on Markowitz (1952) are shown to be optimal. We also check whether the chosen rebalancing period of one year has particular influence on our results. We show that using daily rebalancing, we obtain even better results as when using yearly rebalancing. Lastly, we allow for short-selling, which was constrained in all optimizations before. As expected, mean-variance weights fluctuate even more, resulting in high turnover and transaction costs. The dominance of Minimax over minimum variance portfolios remains.

All results suggest that Minimax strategies provide an attractive alternative asset allocation optimization for a highly risk-averse investor that is concerned with daily risk management. Since Minimax prevents portfolios from realizing high extreme losses, institutional investors that are due to daily risk management can lower their daily portfolio risk. Additionally, Minimax strategies are easily implementable due to its simple algorithm and because exchange traded funds provide easy access to all considered asset classes.

\section{References}

Belousova, J., \& Dorfleitner, G. (2012). On the diversification benefits of commodities from the perspective of 
euro investors. Journal of Banking and Finance, 36, 2455-2472. http://dx.doi.org/10.1016/j.jbankfin.2012.05.003

Britten-Jones, M. (1999). The sampling error in estimates of mean-variance efficient portfolio weights. The Journal of Finance, 54, 655-671. http://dx.doi.org/10.1111/0022-1082.00120

Chateauneuf, A., Maccheroni, F., Marinacci, M., \& Tallon, J. M. (2005). Monotone continuous multiple priors. Economic Theory, 26(4), 973-982. http://dx.doi.org/10.1007/s00199-004-0540-2

DeMiguel, V., Garlappi, L., \& Uppal, R. (2009a). Optimal Versus Naive Diversification: How efficient is the 1/N Portfolio Strategy? Review of Financial Studies, 22(5), 1915-1953. http://dx.doi.org/10.1093/rfs/hhm075

DeMiguel, V., Garlappi, L., Nogales, F. J., \& Uppal, R. (2009b). A generalized approach to portfolio optimization: Improving performance by constraining portfolio norms. Management Science, 55, 798-812. http://dx.doi.org/10.1287/mnsc. 1080.0986

Ding, Y. (2006). Portfolio Selection under Maximum Minimum Criterion. Quality and Quantity, 40(3), 457-468. http://dx.doi.org/10.1007/s11135-005-1054-0

Fan, J., Zhang, J., \& Yu, K. (2012). Vast portfolio selection with gross-exposure constraints. Journal of American Statistical Association, 107, 592-606. http://dx.doi.org/10.1080/01621459.2012.682825

Frost, P., \& Savarino, J. (1988). For better performance: Constrain portfolio weights. Journal of Portfolio Management, 15(1), 29-34. http://dx.doi.org/10.3905/jpm.1988.409181

Garlappi, L., Uppal, R., \& Wang, T. (2007). Portfolio Selection with Parameter and Model Uncertainty: A Multi-Prior Approach. Review of Financial Studies, 20(1), 41-81. http://dx.doi.org/10.1093/rfs/hhl003

Gilboa, I., \& Schmeidler, D. (1989). Maxmin expected utility with non-unique prior. Journal of Mathematical Economics, 18(2), 141-153. http://dx.doi.org/10.1016/0304-4068(89)90018-9

Jagannathan, R., \& Ma, T. (2003). Risk Reduction in Large Portfolios: Why Imposing the Wrong Constraints Helps. Journal of Finance, 58(4), 1651-1684. http://dx.doi.org/10.1111/1540-6261.00580

Kan, R., \& Zhou, G. (2007). Optimal portfolio choice with parameter uncertainty. Journal of Financial and Quantitative Analysis, 42, 621-656. http://dx.doi.org/10.1017/s0022109000004129

Ledoit, O., \& Wolf, M. (2003). Improved estimation of the covariance matrix of stock returns with an application to portfolio selection. Journal of Empirical Finance, 10(5), 603-621. http://dx.doi.org/10.1016/s0927-5398(03)00007-0

Ledoit, O., \& Wolf, M. (2004). A well-conditioned estimator for large-dimensional covariance matrices. Journal of Multivariate Analysis, 88(2), 365-411. http://dx.doi.org/10.1016/s0047-259x(03)00096-4

Ledoit, O., \& Wolf, M. (2008). Robust performance hypothesis testing with the Sharpe ratio. Journal of Empirical Finance, 15(5), 850-859. http://dx.doi.org/10.1016/j.jempfin.2008.03.002

Lintner, J., \& Krasker, W. (1982). Minimax behavior in portfolio selection. Journal of Finance, 37(2), 609-614. http://dx.doi.org/10.1111/j.1540-6261.1982.tb03583.x

Mansini, R., Ogryczak, W., \& Speranza, G. (2003). LP solvable models for portfolio optimization: a classification and computational comparison. IMA Journal of Management Mathematics, 14(3), 187-220. http://dx.doi.org/10.1093/imaman/14.3.187

Markowitz, H. (1952). Portfolio Selection. Journal of Finance, 7(1), 77-91. http://dx.doi.org/10.1111/j.1540-6261.1952.tb01525.x

Merton, R. C. (1980). On estimating the expected return on the market: An exploratory investigation. Journal of Financial Economics, 8(4), 323-361. http://dx.doi.org/10.1016/0304-405x(80)90007-0

Michaud, R. O. (1989). The markowitz optimization enigma: Is 'optimized' optimal? Financial Analysts Journal, 45, 31-42. http://dx.doi.org/10.2469/faj.v45.n1.31

Michaud, R. O. (1998). Efficient Asset Management. Boston, MA: Harvard Business School Press.

Public Fund Survey. (2009). Summary of Findings for FY 2008. Prepared by Keith Brainard. Retrieved from http://www.publicfundsurvey.org

Sengupta, J. (1982). A minimax policy for optimal portfolio choice. International Journal of Systems Science, 13(1), 39-56. http://dx.doi.org/10.1080/00207728208926329

Tütüncü, R., \& Koenig, M. (2004). Robust Asset Allocation. Annals of Operations Research, 132(1-4), 157-187. 
http://dx.doi.org/10.1023/b:anor.0000045281.41041.ed

Young, M. (1998). A Minimax Portfolio Selection Rule with Linear Programming Solution. Management Science, 44(5), 673-683. http://dx.doi.org/10.1287/mnsc.44.5.673

\section{Appendix}

\section{Appendix A. Robustness Check: Yearly Returns}

Table A1. Robustness check: yearly returns

\begin{tabular}{llllll}
\hline PERFORMANCE & Return (\%) & Vola (\%) & Sharpe Ratio & Excess Return & Turnover \\
\hline Minimax & 11.2 & 10.3 & 0.739 & over Minimax (\%) & 8.2 \\
Minimum Variance & 11.9 & 9.3 & 0.900 & +0.7 & 8.9 \\
Equal Weights & 11.7 & 12.4 & 0.653 & +0.5 & 0 \\
Fixed Weights & 11.4 & 12.3 & 0.631 & +0.2 & 0 \\
Mean-Variance & 11.0 & 23.3 & 0.318 & -0.2 & 30 \\
\hline
\end{tabular}

Table A2.

\begin{tabular}{lllll}
\hline RISK & Volatility $(\%)$ & $\operatorname{VaR}(0.95)(\%)$ & $\operatorname{VaR}(0.99)(\%)$ & Max. Loss $(\%)$ \\
\hline Minimax & 10.3 & -8.1 & -10.8 & -10.8 \\
Minimum Variance & 9.3 & -2.4 & -4.3 & -10.0 \\
Equal Weights & 12.4 & -17.6 & -28.3 & -28.3 \\
Fixed Weights & 12.3 & -14.9 & -23.6 & -36.9 \\
Mean-Variance & 23.3 & -37.6 & -45.0 & -45.0 \\
\hline
\end{tabular}

All numbers are based on yearly returns. Return (\%) is calculated geometrically. The Sharpe Ratio is calculated by substracting the yearly risk-free rate from yearly returns, and then dividing by their yearly standard deviation. Turnover is proxied by the sum of absolute deviations in weights before and after rebalancing. We rebalance portfolios every year, using past 250 trading days for our optimizations. VaR stands for Value-at-Risk, which indicates the minimum return (or equivalently, the maximum loss) for a certain significance level $(95 \%$ or $99 \%)$. Short-selling is not allowed for all optimizations. All measures are calculated over the whole period of 1990 to 2010.

\section{Appendix B. Robustness Check: Daily Rebalancing}

Table B1. Robustness check: daily rebalancing

\begin{tabular}{lllllll}
\hline PERFORMANCE & Return (\%) & Vola (\%) & Sharpe Ratio & Excess Return & Turnover & CEV3 \\
\hline Minimax & 0.04 & 0.44 & 0.058 & over Minimax (\%) & 40.4 & 3.7 \\
Minimum Variance & 0.04 & 0.42 & 0.057 & -0.002 & 41.8 & 3.6 \\
Equal Weights & 0.04 & 0.79 & 0.036 & +0.003 & 0 & 3.4 \\
Fixed Weights & 0.04 & 0.77 & 0.036 & +0.002 & 0 & 3.3 \\
Mean-Variance & 0.06 & 0.94 & 0.052 & +0.023 & 394.7 & 4.9 \\
\hline
\end{tabular}

Table B2.

\begin{tabular}{lllll}
\hline RISK & Volatility (\%) & $\operatorname{VaR}(0.95)(\%)$ & $\operatorname{VaR}(0.99)(\%)$ & Max. Loss (\%) \\
\hline Minimax & 0.44 & -0.6 & -1.0 & -2.1 \\
Minimum Variance & 0.42 & -0.5 & -1.1 & -2.6 \\
Equal Weights & 0.79 & -0.9 & -2.3 & -8.7 \\
Fixed Weights & 0.77 & -1.0 & -2.1 & -7.3 \\
Mean-Variance & 0.94 & -1.7 & -3.6 & -16.8 \\
\hline
\end{tabular}

All numbers are based on daily returns. Return (\%) is calculated geometrically. The Sharpe Ratio is calculated by substracting the daily risk-free rate from daily returns, and then dividing by their daily standard deviation. Turnover is proxied by the sum of absolute deviations in weights before and after rebalancing. We rebalance 
portfolios every day, using past 250 trading days for our optimizations. CEV3 is the Certainty Equivalent for a mean-variance investor with risk aversion parameter $\lambda=3$. VaR stands for Value-at-Risk, which indicates the minimum return (or equivalently, the maximum loss) for a certain significance level (95\% or 99\%). Short-selling is not allowed for all optimizations. All measures are calculated over the whole period of 1990 to 2010.

\section{Appendix C. Robustness Check: Short-Selling Allowed}

Table C1. Robustness check: short-selling allowed (daily returns, yearly rebalancing)

\begin{tabular}{lllllll}
\hline PERFORMANCE & Return (\%) & Vola (\%) & Sharpe Ratio & Excess Return & Turnover & CEV3 \\
\hline Minimax & 0.05 & 0.54 & 0.071 & over Minimax (\%) & 9.2 & 4.8 \\
Minimum Variance & 0.04 & 0.40 & 0.063 & -0.013 & 10.2 & 3.8 \\
Mean-Variance & 0.58 & 4.30 & 0.131 & +0.530 & 243.3 & 39.2 \\
\hline
\end{tabular}

Table C2.

\begin{tabular}{lllll}
\hline RISK & Volatility $(\%)$ & $\operatorname{VaR}(0.95)(\%)$ & $\operatorname{VaR}(0.99)(\%)$ & Max. Loss $(\%)$ \\
\hline Minimax & 0.54 & -0.6 & -1.0 & -2.1 \\
Minimum Variance & 0.40 & -0.5 & -1.1 & -2.6 \\
Mean-Variance & 4.30 & -6.0 & -10.7 & -31.9 \\
\hline
\end{tabular}

Table C3.

\begin{tabular}{lllllll}
\hline PERFORMANCE & Return (\%) & Vola (\%) & Sharpe Ratio & Excess Return & Turnover & CEV3 \\
\hline Minimax & 0.04 & 0.44 & 0.058 & over Minimax (\%) & 40.4 & 3.7 \\
Minimum Variance & 0.04 & 0.57 & 0.045 & 0.000 & 46.5 & 3.6 \\
Mean-Variance & 0.21 & 4.90 & 0.040 & +0.170 & 3818.9 & -2.9 \\
\hline
\end{tabular}

Table C4.

\begin{tabular}{lllll}
\hline RISK & Volatility (\%) & $\operatorname{VaR}(0.95)(\%)$ & $\operatorname{VaR}(0.99)(\%)$ & Max. Loss (\%) \\
\hline Minimax & 0.44 & -0.6 & -1.0 & -2.1 \\
Minimum Variance & 0.57 & -0.6 & -1.1 & -14.7 \\
Mean-Variance & 4.90 & -7.0 & -12.7 & -60.3 \\
\hline
\end{tabular}

All numbers are based on daily returns. Return (\%) is calculated geometrically. The Sharpe Ratio is calculated by substracting the daily risk-free rate from daily returns, and then dividing by their daily standard deviation. Turnover is proxied by the sum of absolute deviations in weights before and after rebalancing. We rebalance portfolios every year in Table C3 and every day in Table C4, using past 250 trading days for our optimizations. CEV3 is the Certainty Equivalent for a mean-variance investor with risk aversion parameter $\lambda=3$. VaR stands for Value-at-Risk, which indicates the minimum return (or equivalently, the maximum loss) for a certain significance level $(95 \%$ or $99 \%)$. Short-selling is allowed for all optimizations. All measures are calculated over the whole period of 1990 to 2010 . 


\section{Appendix D. Robustness Check: Crises Periods}

Table D1. Performance in crises

\begin{tabular}{llllllllllllll}
\hline & \multicolumn{3}{c}{ Internet bubble 2000/2001 } & \multicolumn{3}{c}{ Terrorist attack 9/11/2001 } & \multicolumn{3}{c}{ Banking crisis 2007/2008 } & \multicolumn{3}{l}{ Recession 2008-2010 } \\
& CE3 & SR & Max. & CE3 & SR & Max. & CE3 & SR & Max. & CE3 & SR & Max. \\
& & & Loss & & & & Loss & & & Loss & & & Loss \\
\hline Minimax & 8.1 & 0.104 & 1.71 & 3.6 & 0.045 & 1.50 & 3.9 & 0.073 & 1.05 & 4.6 & 0.076 & 2.06 \\
MIV & 7.7 & 0.107 & 1.89 & 3.8 & 0.046 & 1.56 & 3.7 & 0.077 & 1.31 & 4.5 & 0.074 & 2.05 \\
EW & 5.9 & 0.059 & 5.06 & 2.7 & 0.031 & 1.62 & 2.3 & 0.024 & 1.89 & -2.9 & -0.006 & 8.71 \\
FW & 2.6 & 0.029 & 6.01 & 1.7 & 0.015 & 1.78 & 2.6 & 0.029 & 1.93 & -1.7 & -0.004 & 7.31 \\
MV & 8.5 & 0.075 & 4.48 & -4.2 & -0.016 & 3.20 & 10.8 & 0.105 & 3.01 & 7.0 & 0.075 & 3.10 \\
\hline
\end{tabular}

Note. This table reports out-of-sample performance (CE3, SR, Max. Loss in \%) for the Minimax portfolio and its competitors in several crises periods. We analyze the following crises: (1) Internet bubble 2000/2001, (2) Terrorist attack 9/11/2001, (3) Banking crises 2007/2008 and (4) Economic recession 2008-2010.

\section{Copyrights}

Copyright for this article is retained by the author(s), with first publication rights granted to the journal.

This is an open-access article distributed under the terms and conditions of the Creative Commons Attribution license (http://creativecommons.org/licenses/by/3.0/). 